\title{
Classification of chronic cough by systematic treatment cascade trial starting with beta agonist
}

\author{
Hideyasu Shimizu', Masamichi Hayashi', Yuji Saito', Yuki Mieno', Yasuo Takeuchi', Fumihiko Sasaki',
} Hiroki Sakakibara', Kensei Naito ${ }^{2}$ and Mitsushi Okazawa ${ }^{1 *}$

\begin{abstract}
Background: Chronic cough is one of the most challenging symptoms to diagnose and treat, not only because of the variety of underlying disorders but also its varying susceptibility to treatments. Etiological studies of chronic cough vary depending on the clinical settings and the particular interests of investigators.

Objectives: The purposes of this study were first to categorize the etiology of chronic cough by its response to systematic diagnostic treatments starting from the $\beta_{2}$ agonist and second to sub-categorize $\beta_{2}$ agonist responsive cough (BRC) by the airway hyperresponsiveness.

Methods: One hundred and eighty-four never-smokers received the maximal dose of procaterol to diagnose BRC. BRC was sub-categorized into two groups with or without airway hyperresponsiveness measured by the methacholine challenge test. Sinobronchial syndrome (SBS) was diagnosed by postnasal drip symptoms and by the response to clarythromycin and carbocysteine. Atopic cough (AC) was diagnosed by the evidence of atopy and the response to cetirizine hydrochloride. Gastroesophageal reflux disease (GERD) was diagnosed by the response to rabeprazole sodium. Since we did not investigate eosinophil counts in the tissue or in the induced sputum, no diagnosis of eosinophilic bronchitis was made.
\end{abstract}

Results: One hundred and nine patients had BRC. Twenty-three of them had bronchial asthma (BA), 53 had cough variant asthma (CVA) and 33 had non-hyperresponsive BRC (NHBRC). Thirty-one patients had GERD, 27 had AC and 14 had SBS. Twenty-five patients had more than one diagnosis in combination, while 6 had other miscellaneous diseases. Twelve patients were undiagnosed and 11 dropped out of the study.

Conclusions: The majority of chronic cough was BRC. NHBRC was a new chronic cough entity. GERD is a common cause of chronic cough in Japan, as in Western countries. AC and SBS are also causes of chronic cough in Japan.

Trial registration: University hospital medical information network (UMIN 000007483).

Keywords: Airway hyperresponsiveness, $\beta_{2}$ agonist, Bronchial asthma, Cough variant asthma, Non-hyperresponsive and $\beta_{2}$ agonist responsive cough, Gastroesophageal reflex disease, Sinobronchial syndrome, Atopic cough,

Postnasal drip, Chronic cough

\section{Background}

Although cough is the most effective defense mechanism for eliminating foreign materials, including numerous pathogens from the airways, it deteriorates health-related quality of life [1]. Therefore, cough is the most common symptom for which patients seek medical attention [2].

\footnotetext{
* Correspondence: mokazawa@fujita-hu.ac.jp

'Department of Internal Medicine, Division of Respiratory Medicine and Clinical Allergy, Fujita Health University, 1-98 Dengakugakubo Kutsukakecho, Toyoake 470-1192, Japan

Full list of author information is available at the end of the article
}

Chung and Pavord classified the chronic cough into corticosteroid-responsive eosinophilic airway diseases, such as asthma (BA), cough variant asthma (CVA), eosinophilic bronchitis (EB) and corticosteroid resistant disorders such as gastro-esophageal reflux disease (GERD), and the postnasal drip syndrome (PNDS) or rhino-sinusitis [3]. Although all the eosinophilic airway diseases respond to corticosteroid treatment, it is not clear whether these disorders are separate entities, or all one disorder with different levels of severity. Indeed, $\sim 30 \%$ of CVA is reported to develop into BA $[4,5]$,

\section{Biomed Central}


suggesting that some types of CVA could be a precursor of BA. On the other hand, some types of PNDS, such as allergic rhinitis and $\mathrm{AC}$, also respond to corticosteroid [6]. Obviously, the prognosis and impact on healthrelated quality of life from these conditions are quite different from bronchial asthma, and it is not easy to decide how long corticosteroid treatment should be continued. Therefore, investigating how to differentiate corticosteroid-responsive cough from other types of cough is very worthwhile.

Etiological studies of chronic cough vary depending on the clinical setting, the particular interest, the age of patients, and the local definition of diseases [4,7-10]. For example, sinobronchial syndrome (SBS) is more common in Japan, whereas GERD is less common [11].

The purposes of this study were to categorize the etiology of chronic cough by its response to the systematic diagnostic treatments starting from a high dose of an inhaled $\beta_{2}$ agonist, and secondarily to sub-categorize the $\beta_{2}$ agonist responsive cough (BRC) by airway hyperresponsiveness using the methacholine challenge test.

\section{Methods}

This study was approved by the ethics committee of Fujita Health University, and written informed consent was obtained from all the patients who participated. One hundred eighty-four consecutive never-smokers with no chest radiograph abnormalities, who visited the cough clinic of Fujita Health University complaining of chronic cough lasting for more than 8 weeks, were enrolled. Our cough clinic is specialized for patients whose major complaint is chronic cough. More than $90 \%$ of the enrolled patients visited our clinic without referrals from other doctors. Forty-seven were male and 137 were female. The mean age of males and females was $43 \pm$ 14 years and $45 \pm 16$ years, respectively. When a patient was taking angiotensin converting enzyme inhibitor (ACEI) for the treatment of hypertension, the medication was changed. After obtaining a medical history, laboratory examinations including serum IgE levels and methacholine challenge test were performed. The methacholine challenge test was performed according to the guideline [12]. Briefly, the forced expiratory volume in one second $\left(\mathrm{FEV}_{1}\right)$ was measured after 2 minutes' inhalation of saline and then the doubling dose of methacholine was started from $0.031 \mathrm{mg} / \mathrm{ml}$. If the final doubling dose of $16 \mathrm{mg} / \mathrm{ml}$ was reached, a higher dose of $20 \mathrm{mg} / \mathrm{ml}$ was added in this study. The methacholine challenge test was diagnosed to be positive, when $\mathrm{FEV}_{1}$ decreased by more than $20 \%$ after inhalation of an increasing concentration of methacholine $\left(\mathrm{PC}_{20}\right)$ of $8 \mathrm{mg} / \mathrm{ml}$ or less. Then, the stepwise diagnostic treatments were applied (Figure 1). In each step, the patient was subjectively classified as a responder (RD) when the cough completely diminished. The patient was classified as a partial-responder (PR) when the cough had clearly decreased, yet persisted even with the treatment. The patient was classified as a non-responder (NR) when the cough did not decrease at all by the treatment. In step 1 , all the patients received a metered-dose short-acting $\beta_{2}$ agonist inhalation to start with. They received two puffs of procaterol $(20 \mu \mathrm{g}), 4$ times per day for 5 days using an procaterol inhaler (Meptin Air Inhaler ${ }^{\mathrm{R}}$, Otsuka Pharmaceutical Co. Ltd., Japan). This is the maximal daily dose for Japanese, and all the patients were compliant with the protocol, although some experienced tremor or palpitation. Patients who responded to procaterol by the end of the $5^{\text {th }}$ day underwent an airway reversibility test 24 hours after cessation of the procaterol inhaler. The reversibility test was performed according to the guideline [13]. Briefly, forced expiratory maneuvers were repeated before and 15 minutes after nebulization of salbutamol solution $(0.65 \mathrm{mg} / \mathrm{ml})$ for 5 minutes. The reversibility test was considered positive when $\mathrm{FEV}_{1}$ increased by more than $12 \%$ and $200 \mathrm{ml}$ from the baseline values. Thereafter, patients who responded to procaterol received an inhaled corticosteroid (ICS), either fluticasone propionate $(400 \mu \mathrm{g})$, budesonide $(800 \mu \mathrm{g})$ or beclomethasone dipropionate $(400 \mu \mathrm{g})$ throughout the protocol. The final assessment of step 1 was made 2 weeks after inhalation of ICS. PRs at step 1 who had postnasal drip (PND) and phlegm received step 2-a treatment of oral administration of $400 \mathrm{mg}$ clarithromycin and $1500 \mathrm{mg}$ carbocysteine per day for 14 days in addition to ICS. PRs at step2-a received step 3 treatment of oral administration of $20 \mathrm{mg}$ rabeprazole sodium per day for 14 days on top of ICS and step 2-a treatment. PRs at step1 who did not have PND and phlegm skipped both step 2 treatments and received step 3 treatment of oral administration of $20 \mathrm{mg}$ rabeprazole sodium per day on top of ICS. For NRs at step 1 who had a previous history of allergic diseases, increased serum total IgE levels $(>130 \mathrm{IU} / \mathrm{ml})$ or specific IgE antibodies measured by ELISA method (MAST26, Hitachi Chemical Co. Ltd., Tokyo), ICS was not used, and the step 2-b treatment of oral administration of $10 \mathrm{mg}$ cetirizine hydrochloride per day for 14 days was applied. NRs at step 1 who had PND and phlegm received step 2-a treatments. NRs both to step 2-a and 2-b treatments proceeded to step 3 treatment in which $20 \mathrm{mg}$ of rabeprazole sodium alone was used per day for 14 days.

\section{Diagnostic criteria}

RDs and PRs at step 1 were diagnosed with a $\beta_{2}$ agonist responsive cough (BRC). BRC patients who had $\mathrm{PC}_{20}$ of $8 \mathrm{mg} / \mathrm{ml}$ or less were classified as having hyperresponsive and $\beta_{2}$ agonist responsive cough (HBRC). Among HBRC, patients with a previous history of bronchial 


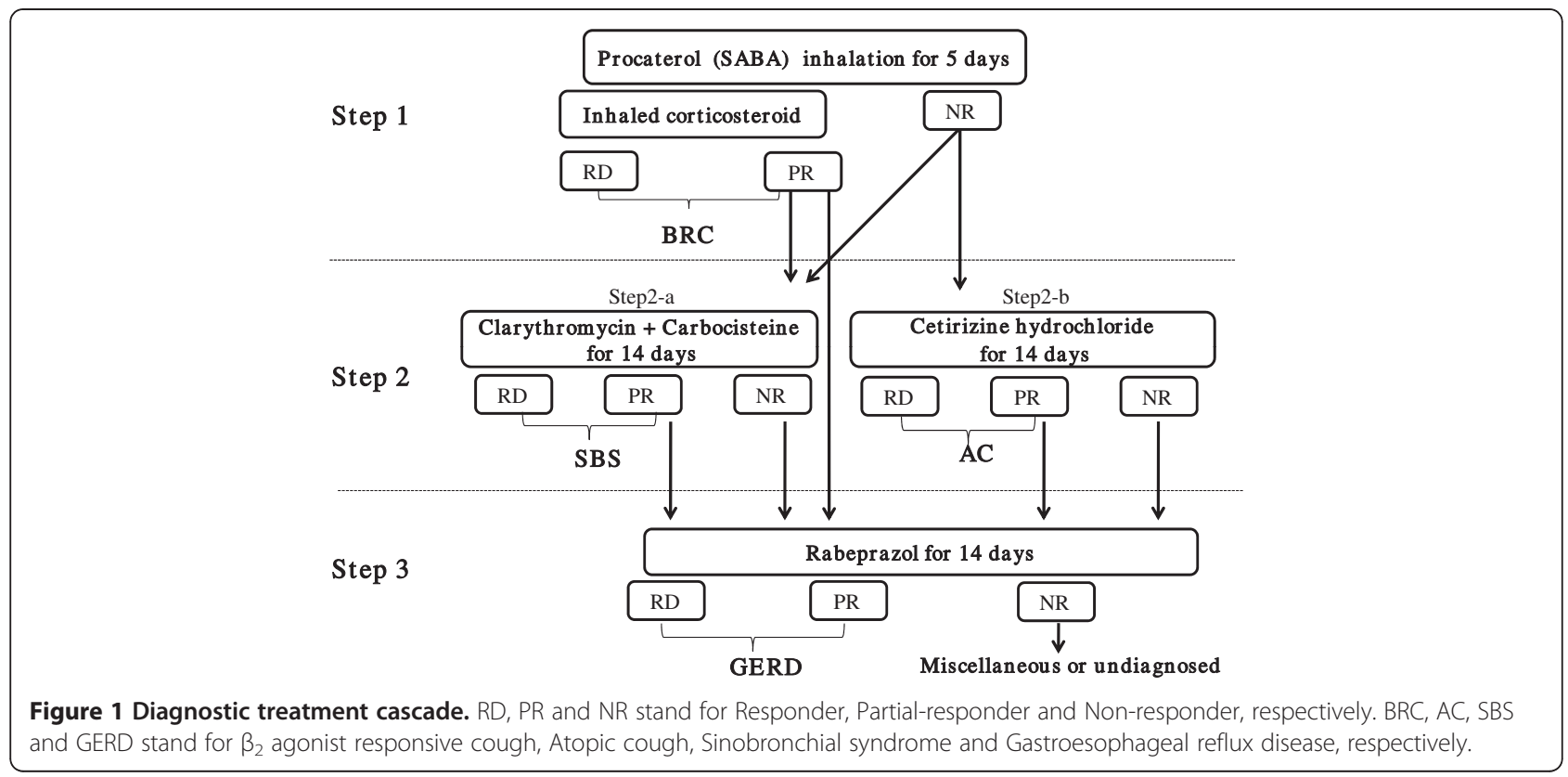

asthma or asthmatic symptoms such as episodic wheeze or dyspnea were categorized with bronchial asthma (BA). Patients who had a positive methacholine challenge test without any asthmatic symptoms such as wheezing or dyspnea were diagnosed with cough variant asthma (CVA). Patients without any asthmatic symptoms who failed to undergo the methacholine challenge test were also categorized as CVA. Patients were diagnosed to have non-hyperresponsive BRC (NHBRC) when they responded to the $\beta_{2}$ agonist but their $\mathrm{PC}_{20}$ was $20 \mathrm{mg} / \mathrm{ml}$ or over. Patients with postnasal drip (PND) and phlegm were diagnosed with sinobronchial syndrome (SBS) [9] when they responded to the oral administration of clarithromycin and carbocysteine. Patients were diagnosed as atopic cough (AC) [9] when they had either a high level of serum IgE (>130 IU/ml), or specific IgE antibodies measured by the ELISA method, or other allergic disorders such as allergic rhinitis or food allergy, and responded to oral administration of cetirizine hydrochloride but not to inhaled $\beta_{2}$ agonist. Patients were diagnosed with gastroesophageal reflux disease (GERD) when they responded to the oral administration of rabeprazole sodium. NRs to all the treatments at step 1-3 received further examinations such as computed tomography, bronchoscopy, sputum bacteriologies, or serological examinations.

\section{Statistics}

RDs in step 1-3 were selected for a basic comparison. The duration of cough, serum IgE level, a symptom of $\mathrm{PND}, \mathrm{PC}_{20}, \% \mathrm{VC}$, and $\% \mathrm{FEV}_{1} / \mathrm{FVC}$ ratio, and flow volume parameters were compared using ANOVA and post hoc test with Bonferroni correction. Changes in the flow volume parameters of BRCs before and after the airway reversibility test were compared using paired $t$-test. The magnitude of change in flow volume parameters were compared using ANOVA and post hoc test with Bonferroni correction. A p value less than 0.05 was considered to be significant. All the statistics were performed using StatView5.0 (HULINKS Inc. Tokyo).

\section{Results}

\section{Classification of chronic cough}

Eleven patients dropped out of the analysis because they did not complete the protocol. One-hundred and nine patients were with BRC (Table 1), and eighty-six of them received methacholine challenge tests. The others did not undergo the tests because of increasing cough during a test or their refusal to take the examination. No patients showed worsening of cough after switching from SABA to ICS in step 1 procedure. Twenty-three patients had a previous history of bronchial asthma, mostly during childhood, or had asthmatic symptoms such as wheezing and were diagnosed with BA. Nineteen of them experienced complete diminution of chronic cough after step 1 treatment but the other 4 patients required step 2 or 3 treatments in addition to step 1 treatment. They were diagnosed with $\mathrm{BA}+\mathrm{SBS}$ or BA + GERD. Fifty-three patients were diagnosed with CVA. Forty-seven of them experienced complete diminution of chronic cough after step 1 treatment but the other 6 patients were diagnosed either with CVA + SBS or with CVA + GERD. Thirty-three patients were diagnosed with NHBRC. Thirty of them experienced complete diminution by step 1 treatment, while the other three were diagnosed either with NHBRC + SBS or NHBRC + GERD. 
Table 1 Classification of BRC

\begin{tabular}{|c|c|c|c|c|c|}
\hline \multirow[b]{2}{*}{ BA } & \multirow[b]{2}{*}{$23(6 / 17)$} & \multicolumn{2}{|c|}{ BRC $109(21 / 88)$} & \multirow[b]{2}{*}{ NHBRC } & \multirow[b]{2}{*}{$33(7 / 26$} \\
\hline & & CVA & $53(8 / 45)$ & & \\
\hline BA & $19(5 / 14)$ & CVA & $47(8 / 39)$ & NHBRC & $30(7 / 23)$ \\
\hline$B A+S B S$ & $2(0 / 2)$ & $C V A+S B S$ & $2(0 / 2)$ & $\mathrm{NHBRC}+\mathrm{SBS}$ & $2(0 / 2)$ \\
\hline $\mathrm{BA}+\mathrm{GERD}$ & $2(1 / 1)$ & $C V A+G E R D$ & $4(0 / 4)$ & $\mathrm{NHBRC}+\mathrm{GERD}$ & $1(0 / 1)$ \\
\hline
\end{tabular}

$B A$, Bronchial asthma; CVA, Cough variant asthma; NHBRC, Non-hyperresponsive and $\beta_{2}$ agonist responsive cough; numbers within parentheses indicate males and females.

Sixty-four patients were diagnosed with non-BRC (Table 2). Among them, thirty-three received methacholine challenge tests. The others did not undergo the tests because of increasing cough during a test or their refusal to take the examination. Twenty-seven of 64 patients responded to the treatment of step 2-b and were diagnosed with AC. The cough completely diminished in 17 of them. Others were diagnosed either with AC + SBS + GERD or AC + GERD. Seven patients were diagnosed with SBS because they responded to the treatment of step 2-a. Two of them required step 3 treatment before their cough diminished and were diagnosed with SBS + GERD. Twelve patients were diagnosed with GERD because their cough completely diminished only by step 3 treatment. Six patients were diagnosed as Miscellaneous (one ACEI-induced cough, 2 non-tuberculous mycobacterial infections, one nocardial bronchitis, one pertussis, and one interstitial pneumonitis).

The RDs who had a single diagnosis at steps $1-3$ were selected for basic comparison (Table 3). Although the duration of cough tended to be shorter in GERD patients, there was no significant difference between groups. Fifteen patients with BA, 26 with CVA, and 10 with NHBRC were atopic. There was no significant difference in serum IgE levels between these groups. A PND symptom was significantly more prevalent in patients with SBS than in other groups. $\mathrm{PC}_{20}$ was significantly lower in BA and CVA than in the other groups. There was no significant difference in $\% \mathrm{VC}$. The $\% \mathrm{FEV}_{1} / \mathrm{FVC}$ ratio was lower in BA than in $\mathrm{AC}$, CVA and NHBRC.

\section{Functional differences in BRC}

Among 96 RDs at step 1, 13 BAs, 10 CVAs and 19 NHBRCs received the airway reversibility test. Although baseline PEFR and FEFR $50 \%$ tended to be lower in CVAs, there were no significant differences between groups (Table 4). PEFR increased significantly after inhalation of salbutamol in BA and CVA patients, but not in NHBRC patients. FEFR $50 \%$ and $\mathrm{FEFR}_{25 \%}$ significantly increased after inhalation of salbutamol in all BRC groups. The increase of PEFR of BA patients was significantly larger than that of NHBRC (Figure 2). There was no significant difference in the increase of $\mathrm{FEFR}_{50 \%}$ and $\mathrm{FEFR}_{25 \%}$ between groups (Figure 2).

\section{Discussion}

In the current study, we found that chronic cough diminished partially or completely in $59 \%$ of the patients who used inhaled $\beta_{2}$ agonist followed by ICS. The percentage of BRC was higher than that in the previous literature $[11,14]$, probably because we used the bronchodilator with a higher dose. CVA was the major cause of chronic cough in our study, as previously reported in Japan $[15,16]$ although this observation is quite different from those in Western countries [17]. In the current study, on the other hand, there was a distinctly different group of patients who responded to beta agonist but did not have airway hyperresponsiveness measured by the methacholine challenge test. We named this condition NHBRC, and it consisted of a significantly large part of BRC (30\%) with a higher prevalence in women (Table 1). Fujimoto et al. reported a similar phenomenon in a limited number of patients who responded to a beta agonist without airway hyperresponsiveness [18]. Although the airway reversibility test estimated by the change in $\mathrm{FEV}_{1}$ was not positive in all BRC patients, there was a difference in the flow volume parameters among BA, CVA and NHBRC. A small but significant increase was observed in PEFR after inhalation of salbutamol in BA and CVA, but not in NHBRC (Table 4). There was a significant

Table 2 Classification of non-BRC

\begin{tabular}{|c|c|c|c|c|c|c|c|}
\hline \multirow[b]{2}{*}{$A C$} & \multirow[b]{2}{*}{$27(5 / 22)$} & \multicolumn{2}{|c|}{ Non-BRC 64 (20/44) } & & & & \\
\hline & & SBS & $7(4 / 3)$ & & & & \\
\hline AC & $17(4 / 13)$ & SBS & $5(3 / 2)$ & GERD & $12(4 / 8)$ & Miscellaneous & $6(0 / 6)$ \\
\hline$A C+S B S+G E R D$ & $1(01)$ & $\mathrm{SBS}+\mathrm{GERD}$ & $2(1 / 1)$ & & & Undiagnosed & $12(7 / 5)$ \\
\hline$A C+G E R D$ & $9(1 / 8)$ & & & & & & \\
\hline
\end{tabular}

$A C$, Atopic cough; SBS, Sinobronchial syndrome; GERD, Gastroesophageal reflux disease; numbers within parentheses indicate males and females. 
Table 3 Basic data

\begin{tabular}{|c|c|c|c|c|c|c|}
\hline & $\begin{array}{c}\text { BA } \\
(n=19)\end{array}$ & $\begin{array}{c}\text { CVA } \\
(n=47)\end{array}$ & $\begin{array}{l}\text { NHBRC } \\
(n=30)\end{array}$ & $\begin{array}{c}\text { AC } \\
(n=17)\end{array}$ & $\begin{array}{c}\text { SBS } \\
(n=5)\end{array}$ & $\begin{array}{c}\text { GERD } \\
(n=12)\end{array}$ \\
\hline Duration (days) & 421 (898) & 195 (428) & 491 (1194) & $654(1406)$ & 399 (396) & $97(39)$ \\
\hline $\operatorname{lgE}(\mathrm{IU} / \mathrm{ml})$ & 1181 (3349) & $291(583)$ & $262(542)$ & 255 (383) & 127 (198) & $139(168)$ \\
\hline PND (\%) & 26 & 32 & 18 & 29 & $100^{*}$ & 17 \\
\hline $\mathrm{PC}_{20}(\mathrm{mg} / \mathrm{ml})$ & $4.347(5.820)^{*}$ & $4.149(2.944)^{*}$ & $>20$ & $>20$ & $>20$ & $>20$ \\
\hline \%VC (\%) & $113(18)$ & $106(15)$ & $112(13)$ & $118(17)$ & $105(20)$ & $115(13)$ \\
\hline$\% \mathrm{FEV}_{1} / \mathrm{FVC}(\%)$ & $72(11)^{*}$ & $81(11)$ & $83(7)$ & $83(7)$ & $75(8)$ & $82(6)$ \\
\hline
\end{tabular}

Data are means (SD). Patients with a single diagnosis were selected for comparison. IgE, serum lgE level; PND (\%), percentage of patients with postnasal drip; $P C_{20}$ methacholine concentration which induces a $20 \%$ decrease in the forced expiratory volume in one second $\left(\mathrm{FEV}_{1}\right)$; \% $\%$, percentage of predicted value of vital capacity; \%FEV $1 / \mathrm{FVC}_{;} \mathrm{FEV}_{1} / \mathrm{FVC}$ ratio expressed by percentage. ${ }^{*} \mathrm{p}<0.05$.

difference in the magnitude of increase in PEFR between BA and NHBRC (Figure 2). These results suggest that bronchoconstriction or increased smooth muscle tone seem to occur less in NHBRC than in the other BRCs. On the other hand, $\mathrm{FEFR}_{50 \%}$ and $\mathrm{FEFR}_{25 \%}$, which are used for estimating flow limitation in the peripheral airways, were significantly increased after salbutamol inhalation in all $\mathrm{BRC}$, and the magnitude of increase among BA, CVA and NHBRC was comparable. Since cough diminished after procaterol inhalation in these patients with $\mathrm{BRC}$, bronchoconstriction of peripheral airways may be the common cause of chronic cough. Although bronchoconstriction does not have a direct effect on the sensitivity of the cough receptor in healthy subjects [19], bronchoconstriction or increased bronchial tone itself may stimulate the afferent tussive nerve, possibly by deformation of the airway epithelium in the peripheral airways [20]. Airway remodeling in BA and CVA has been documented [21], and similar remodeling could be involved in NHBRC inducing exaggerated airway wall deformation during bronchoconstriction so as to cause chronic cough.

Takemura et al., who compared classical BA and CVA, reported that $15 \%$ of CVA patients developed BA after two year observation and that these patients had a higher IgE level than CVA patients who did not develop BA [22]. Their results indicated that the severity of atopic status may be associated with the development of classical asthma with wheezing. Since patients with NHBRC had a significantly higher IgE level as with BA and CVA, it is possible that they could develop CVA or even BA in the future.
As shown in Table 1, there was no combination of diagnoses between BRC and AC. Since ICS was used for BRC in step 1 treatment, and ICS was also effective for AC preventing this combination. Even so, the number of AC patients in our study was far less than was reported by Fujimura et al. [11]. According to the diagnostic criteria of $\mathrm{AC}$ [9], patients with $\mathrm{AC}$ do not respond to beta agonist. The dose of beta agonist for diagnosis, however, is not defined. In the current study, twenty out of 33 NHBRC patients were atopic. If the patients with atopic NHBRC in our study were undertreated with $\beta_{2}$ agonists, they could well have been diagnosed as AC. Then, the percentage of $\mathrm{AC}$ would have become approximately $27 \%$, which is close to the percentage of AC in the previous study by Fujimura et al. [11]. Non-asthmatic eosinophilic bronchitis (NAEB), which shares similar clinical characteristics with $\mathrm{AC}$, is one of the common causes of chronic cough [23]. NAEB is also similar to NHBRC with respect to the effectiveness of ICS and the lack of airway hyperresponsiveness. Since we did not measure the eosinophil counts, it is difficult to speculate whether eosinophilic airway inflammation was involved in NHBRC. On the other hand, the response to betaagonist has not systematically been investigated in patients with NAEB. Therefore, it seems to be very important to investigate whether there are overlaps between NAEB, AC and NHBRC, since a part of patients with NAEB were reported to develop more serious conditions such as BA or airflow limitation during the few years of follow up [24].

Table 4 Flow volume parameters

\begin{tabular}{|c|c|c|c|c|c|c|}
\hline & \multicolumn{2}{|c|}{ BA } & \multicolumn{2}{|c|}{ CVA } & \multicolumn{2}{|c|}{ NHBRC } \\
\hline & Before & After & Before & After & Before & After \\
\hline PEFR & $7.51(2.39)$ & $7.79(2.37)^{*}$ & $6.24(1.02)$ & $6.48(1.27)^{*}$ & $7.67(1.87)$ & $7.72(1.61)^{\mathrm{NS}}$ \\
\hline $\mathrm{FEFR}_{50 \%}$ & $2.85(1.71)$ & $3.33(1.93)^{*}$ & $2.81(0.54)$ & $3.05(0.80)^{*}$ & $3.01(0.66)$ & $3.24(0.64)^{*}$ \\
\hline $\mathrm{FEFR}_{25 \%}$ & $0.80(0.76)$ & $0.92(0.77)^{*}$ & $0.80(0.54)$ & $1.04(0.80)^{*}$ & $0.87(0.46)$ & $1.03(0.50)^{*}$ \\
\hline
\end{tabular}

Data are means (SD). PEFR, peak expiratory flow rate; $F E F R_{50 \%}$, forced expiratory flow rate at $50 \%$ of vital capacity; $F E F R_{25 \%}$, forced expiratory flow rate at $25 \%$ of vital capacity. ${ }^{*} \mathrm{p}<0.05$, NS, not significant. 


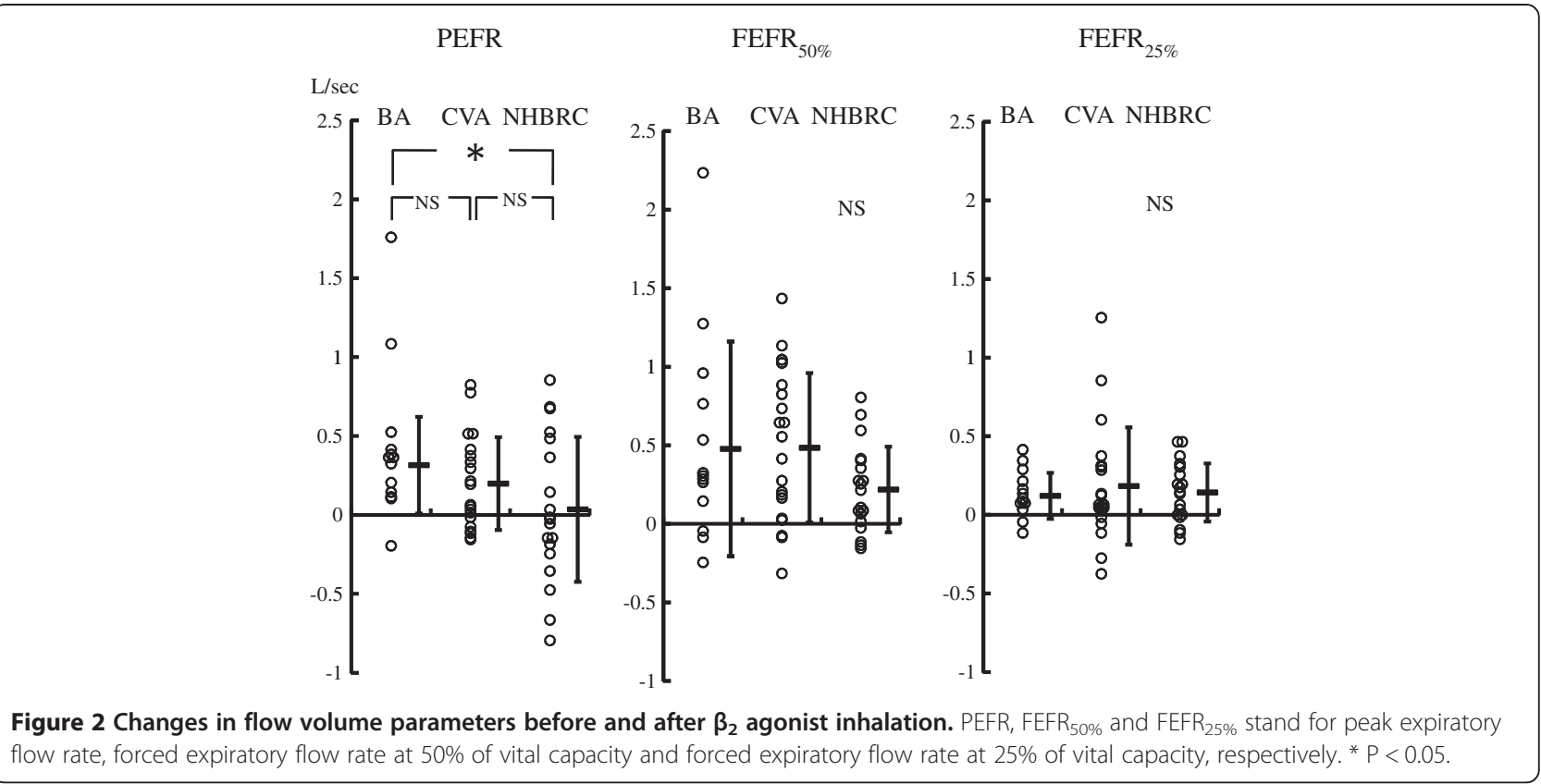

Postnasal drip syndrome has been recognized as one of the major causes of chronic cough [25]. The guideline issued by the American College of Chest Physicians suggested the use of the term "Upper airway cough syndrome (UACS)" instead of PND [26]. In the current study, we did not categorize the patients as UACS since most of the cough was diagnosed otherwise by the systematic treatment cascade. Although PND was observed in $17-32 \%$ of $\mathrm{BA}, \mathrm{CVA}, \mathrm{NHBRC}, \mathrm{AC}$, and GERD patients (Table 3), the cough diminished without H1antagonist in all groups except for AC. Five out of the 17 ACs had PND (Table 3) and could be diagnosed as having UACS, since both UACS and AC respond to the H1-antagonist, suggesting that there could be an overlap between UACS and AC. Moreover, it is not clear that PND itself is the independent cause of chronic cough since PND from allergic rhinitis or rhinosinusitis is frequently associated with airway disorders such as asthma $[27,28]$, and the cough diminishes after treatment of asthma. O'Hara and Jones suggested that PND due to rhinosinusitis without a coexistent chest disease is not a predominant cause of chronic cough [29]. In the current study, all the patients with SBS had PND, as well as a productive cough with phlegm, and responded well to the treatment using clarithromycin and carbocysteine suggesting lower airway involvement. Kohno et al. classified the lower airway involvement in SBS into three categories; chronic bronchitis, bronchiectasis, and diffuse panbronchiolitis [9]. Although it is not known why there are more SBS patients in Japan compared with Western countries, the genetic factors may partially contribute to the discrepancy [30].
GERD is one of the most common causes of chronic cough and is reported to cause up to $41 \%$ of it [25]. However, Fujimura et al. reported that only $2 \%$ of chronic cough was caused by GERD in Japan [11]. In the current study, $6.5 \%$ of chronic cough was caused by GERD alone, and $10.9 \%$ of patients had GERD in combination with other disorders (Tables 1 and 2). This result shows that the percentage of GERD is altogether similar to the results in the literature from Western countries [25]. The prevalence of GERD in Japan is increasing since the end of the 1990s [31,32], possibly due to the change in dietary style and to the decrease in Helicobacter pylori infection brought about by antibiotics treatment. Therefore, the discrepancy between the previous study by Fujimura et al. [11] and ours could be due to the increasing occurrence of GERD, since our study period was several years later than theirs.

\section{Conclusion}

In the current study, we started by diagnosing chronic cough with a bronchodilator. This method is inexpensive and effective for categorizing the etiology of chronic cough. The majority of patients with chronic cough had BRC. NHBRC, a new chronic cough entity, was the major cause of BRC. GERD is a common cause of chronic cough in Japan, as it is in Western countries. AC and SBS are also the causes of chronic cough in Japan.

\section{Abbreviations}

BRC: $\beta_{2}$ agonist responsive cough; HBRC: Hyperresponsive and $\beta_{2}$ agonist responsive cough; NHBRC: Non-hyperresponsive and $\beta_{2}$ agonist responsive cough; BA: Bronchial asthma; CVA: Cough variant asthma; 
GERD: Gastroesophageal reflux disease; SBS: Sinobronchial syndrome; AC: Atopic cough; PND: Postnasal drip; UACS: Upper airway cough syndrome; PPI: Proton pump inhibitor; RD: Responder; PR: Partial-responder; NR: Non-responder; ICS: Inhaled corticosteroid; ACEl: Angiotensin converting enzyme inhibitor.

\section{Competing interests}

This study was supported by the research fund of Fujita Health University, which served to cover the costs of transportation and hotel accommodations for the Conference and the financing of manuscripts, including the article processing charge. There are no competing interest with the university or any other organizations which are related to the patients who participated in this study.

\section{Authors' contributions}

$\mathrm{HS}$ designed the study and wrote the first draft under the supervision of $\mathrm{MO}$. $\mathrm{MH}, \mathrm{YS}, \mathrm{YM}, \mathrm{YT}, \mathrm{FS}$ and HS participated in the study by recruiting the patients, and by giving suggestions for the first draft. KN participated in the study by diagnosing sinobronchitis from a specialist's point of view as an otolaryngologist. All authors have read and approved the final manuscript.

\section{Acknowledgements}

The authors are grateful to Professor Shuji Hashimoto for his generous assistance with statistical analysis in this study. We are also especially appreciative of Professor Peter D. Paré for his helpful suggestions and support in completing the manuscript.

\section{Author details}

${ }^{1}$ Department of Internal Medicine, Division of Respiratory Medicine and Clinical Allergy, Fujita Health University, 1-98 Dengakugakubo Kutsukakecho, Toyoake 470-1192, Japan. ²Department of Otolaryngology, Fujita Health University, Toyoake 470-1192, Japan.

Received: 30 March 2012 Accepted: 3 February 2013

Published: 7 February 2013

\section{References}

1. Braido F, Baiardini I, Tarantini F, Fassio O, Balestracci S, Pasquali M, Tarchino F, Canonica GW: Chronic cough and QoL in allergic and respiratory diseases measured by a new specific validated tool-CCIQ. J Investig Allergol Clin Immunol 2006, 16(2):110-116.

2. Schappert SM, Burt CW: Ambulatory care visits to physician offices, hospital outpatient departments, and emergency departments: United States, 2001-02. Vital Health Stat 2006, 13(159):1-66.

3. Chung KF, Pavord ID: Prevalence, pathogenesis, and causes of chronic cough. Lancet 2008, 371(9621):1364-1374.

4. Fujimura M, Ogawa $H$, Nishizawa $Y$, Nishi K: Comparison of atopic cough with cough variant asthma: is atopic cough a precursor of asthma? Thorax 2003, 58(1):14-18

5. Matsumoto H, Niimi A, Takemura M, Ueda T, Tabuena R, Yamaguchi M, Matsuoka H, Hirai T, Muro S, Ito $Y$, et al: Prognosis of cough variant asthma: a retrospective analysis. J Asthma 2006, 43(2):131-135.

6. Fujimura M, Ogawa H, Yasui M, Matsuda T: Eosinophilic tracheobronchitis and airway cough hypersensitivity in chronic non-productive cough. Clin Exp Allergy 2000, 30(1):41-47.

7. Chang $A B$, Glomb WB: Guidelines for evaluating chronic cough in pediatrics: ACCP evidence-based clinical practice guidelines. Chest 2006 129(1 Suppl):260S-283S.

8. Gibson PG, Dolovich J, Denburg J, Ramsdale EH, Hargreave FE: Chronic cough: eosinophilic bronchitis without asthma. Lancet 1989, 1(8651):1346-1348.

9. Kohno S, Ishida T, Uchida Y, Kishimoto H, Sasaki H, Shioya T, Tokuyama K, Niimi A, Nishi K, Fujimura M, et al: The Japanese Respiratory Society guidelines for management of cough. Respirology 2006, 11(Suppl 4):S135-S186

10. McGarvey LP: Cough. 6: Which investigations are most useful in the diagnosis of chronic cough? Thorax 2004, 59(4):342-346.

11. Fujimura M, Abo M, Ogawa $H$, Nishi $K$, Kibe $Y$, Hirose $T$, Nakatsumi $Y$, Iwasa $\mathrm{K}$ : Importance of atopic cough, cough variant asthma and sinobronchial syndrome as causes of chronic cough in the Hokuriku area of Japan. Respirology 2005, 10(2):201-207.
12. Crapo RO, Casaburi $R$, Coates $A L$, Enright $P L$, Hankinson JL, Irvin CG, MacIntyre NR, McKay RT, Wanger JS, Anderson SD, et al: Guidelines for methacholine and exercise challenge testing-1999. This official statement of the American Thoracic Society was adopted by the ATS Board of Directors, July 1999. Am J Respir Crit Care Med 2000, 161(1):309-329.

13. Pellegrino R, Viegi G, Brusasco V, Crapo RO, Burgos F, Casaburi R, Coates A, van der Grinten CP, Gustafsson P, Hankinson J, et al: Interpretative strategies for lung function tests. Eur Respir J 2005, 26(5):948-968.

14. Morice AH, Fontana GA, Sovijarvi AR, Pistolesi M, Chung KF, Widdicombe J, O'Connell F, Geppetti P, Gronke L, De Jongste J, et al: The diagnosis and management of chronic cough. Eur Respir J 2004, 24(3):481-492.

15. Johnson D, Osborn LM: Cough variant asthma: a review of the clinical literature. J Asthma 1991, 28(2):85-90.

16. Shirahata K, Fujimoto K, Arioka H, Shouda R, Kudo K, Ikeda S: Prevalence and clinical features of cough variant asthma in a general internal medicine outpatient clinic in Japan. Respirology 2005, 10(3):354-358.

17. Niimi A: Geography and cough aetiology. Pulm Pharmacol Ther 2007, 20(4):383-387.

18. Fujimoto K, Yamaguchi S, Urushibata K, Koizumi T, Kubo K: Sputum eosinophilia and bronchial responsiveness in patients with chronic nonproductive cough responsive to anti-asthma therapy. Respirology 2003, 8(2):168-174.

19. Fujimura M, Sakamoto S, Kamio Y, Matsuda T: Effects of methacholine induced bronchoconstriction and procaterol induced bronchodilation on cough receptor sensitivity to inhaled capsaicin and tartaric acid. Thorax 1992, 47(6):441-445.

20. Karlsson JA, Sant'Ambrogio G, Widdicombe J: Afferent neural pathways in cough and reflex bronchoconstriction. J Appl Physiol 1988, 65(3):1007-1023.

21. Niimi A, Matsumoto H, Minakuchi M, Kitaichi M, Amitani R: Airway remodelling in cough-variant asthma. Lancet 2000, 356(9229):564-565.

22. Takemura M, Niimi A, Matsumoto H, Ueda T, Yamaguchi M, Matsuoka $H$, Jinnai M, Chin K, Mishima M: Atopic features of cough variant asthma and classic asthma with wheezing. Clin Exp Allergy 2007, 37(12):1833-1839.

23. Brightling CE: Chronic cough due to nonasthmatic eosinophilic bronchitis: ACCP evidence-based clinical practice guidelines. Chest 2006 129(1 Suppl):116S-121S.

24. Berry MA, Hargadon B, McKenna S, Shaw D, Green RH, Brightling CE, Wardlaw AJ, Pavord ID: Observational study of the natural history of eosinophilic bronchitis. Clin Exp Allergy 2005, 35(5):598-601.

25. Palombini BC, Villanova CA, Araujo E, Gastal OL, Alt DC, Stolz DP, Palombini CO: A pathogenic triad in chronic cough: asthma, postnasal drip syndrome, and gastroesophageal reflux disease. Chest 1999, 116(2):279-284.

26. Irwin RS, Baumann MH, Bolser DC, Boulet LP, Braman SS, Brightling CE, Brown KK, Canning BJ, Chang AB, Dicpinigaitis PV, et al: Diagnosis and management of cough executive summary: ACCP evidence-based clinical practice guidelines. Chest 2006, 129(1 Suppl):1S-23S.

27. Bresciani M, Paradis L, Des Roches A, Vernhet H, Vachier I, Godard P Bousquet J, Chanez P: Rhinosinusitis in severe asthma. J Allergy Clin Immunol 2001, 107(1):73-80.

28. Grossman J: One airway, one disease. Chest 1997, 111(2 Suppl):11S-16S

29. O'Hara J, Jones NS: The aetiology of chronic cough: a review of current theories for the otorhinolaryngologist. J Laryngol Otol 2005, 119(7):507-514.

30. Matsuzaka Y, Tounai K, Denda A, Tomizawa M, Makino S, Okamoto K, Keicho N, Oka A, Kulski JK, Tamiya G, et al: Identification of novel candidate genes in the diffuse panbronchiolitis critical region of the class I human MHC. Immunogenetics 2002, 54(5):301-309.

31. Fujiwara Y, Arakawa T: Epidemiology and clinical characteristics of GERD in the Japanese population. J Gastroenterol 2009, 44(6):518-534.

32. Mishima I, Adachi K, Arima N, Amano K, Takashima T, Moritani M, Furuta K, Kinoshita $Y$ : Prevalence of endoscopically negative and positive gastroesophageal reflux disease in the Japanese. Scand J Gastroenterol 2005, 40(9):1005-1009.

doi:10.1186/1745-9974-9-4

Cite this article as: Shimizu et al.: Classification of chronic cough by systematic treatment cascade trial starting with beta agonist. Cough 2013 9:4. 